\title{
Earthquake Insurance, Catastrophe Insurance and Earthquake Risk \& Loss: a Comparative Analysis Based on CiteSpace
}

\author{
Cong Lang ${ }^{1}$, Xinyan $\mathrm{Wu}^{1,} *$, Haoyu $\mathrm{Wu}^{2}$ \\ ${ }^{1}$ Institute of Geophysics, China Earthquake Administration, Beijing 100081, China \\ ${ }^{2}$ Shanxi Earthquake Agency, Taiyuan 030021, China
}

Received October 1, 2018

Accepted October 25, 2018

\begin{abstract}
China suffers severe earthquake disasters. Earthquake insurance can effective transfer and disperse earthquake risk. This paper compared the keyword cluster and institution network of earthquake insurance, other catastrophe insurance and earthquake risk \& loss. The keyword cluster showed that earthquake insurance and other catastrophe study relied on non-engineering approach, such as institutions, policy and risk management. While study about earthquake risk \& loss mainly relied on engineering approach. Besides, the institution network of earthquake insurance and earthquake risk \& loss indicated that there was a lack of institutional cooperation and gaps between natural science and social science. As earthquake insurance is a multidiscipline reform of risk management, earthquake insurance study should need multidiscipline studies.
\end{abstract}

Keywords: Earthquake insurance, Catastrophe insurance, Earthquake risk \& loss, CiteSpace, Keyword cluster.

\section{地震保险、巨灾保险和地震风险损失一一基于 CiteSpace 的比较分析 ${ }^{*}$}

\author{
郎 从 $^{1}$, 吴新燕 ${ }^{1, *}$, 吴昊昱 ${ }^{2}$ \\ 1 中国地震局地球物理研究所, 北京 100081 , 中国 \\ 2 山西省地震局, 太原030021, 中国
}

\begin{abstract}
摘 要
中国地震灾害频繁而强烈, 地震保险是常用风险转移工具之一，本文使用 CiteSpace 分析 “地震保 险”、“其他巨灾保险”、“地震风险和损失”三大研究主题的研究文献。关键词共现分析表明, 地震巨 灾保险和其它巨灾保险研究较注重制度等非工程方面的研究, 相关研究较少结合工程技术。而地震风险和 损失研究则多注重工程技术，较少结合制度、风险管理等非工程防御手段。研究机构共现网络分析则表 明, 地震巨灾保险和地震风险和损失研究存在机构交流合作少、学科割裂的现象。因此，应结合工程和非 工程的研究, 进行跨学的巨灾保险和地震风险损失研究。
\end{abstract}

关键词：地震保险，巨灾保险，地震风险和损失，CiteSpace，关键词聚类

\section{1. 引言}

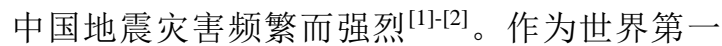
人口大国，第二经济大国，中国面临极高的地震风 险, 根据 GAR2009 报告, 中国具有全球最高的地震
人口死亡风险指数 ${ }^{[3]}$ 。地震给我国造成巨大的损 失, 历史上死亡人口最多的地震就发生在中国,

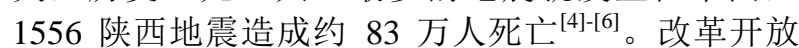
后，地震也给我国带来巨大的人口伤亡和经济损 失， 2008 年汶川地震造成 8 万余人死亡和失踪, 6920 亿元直接经济损失 ${ }^{[7]}$ 。

\footnotetext{
* Corresponding author: wuxy1977@ sina.com
} 
保险是进行巨灾风险转移的手段之一。相比财 政拨款、慈善捐款、国际捐款和灾民自救, 保险可 以克服国家财政拨款有限, 捐赠不可预知的缺点, 在国际上广为应用。

党的十八届三中全会通过的《中共中央关于全 面深化改革若干重大问题的决定》, 明确提出 “完 善保险经济补偿机制, 建立巨灾保险制度”。《国 务院关于加快发展现代保险服务业的若干意见》提 出“建立巨灾保险制度”。2016 年 5 月, 中国保监 会、财政部印发了《建立城乡居民住宅地震巨灾保 险制度实施方案》, 指出要 “结合我国国情, 以地 震巨灾保险为突破口, 探索建立专项巨灾保险制 度”。我国也在 2015 年 8 月, 在云南省大理白族自 治州启动全国首个农房地震保险试点。预计在 3 年 的试点期限内, 为大理州所辖 12 县 (市) 82.43 万 户农村房屋及 356.92 万居民提供风险保障。

目前, 国际上开展较多的巨灾保险包括洪水保 险、台风保险和地震保险 ${ }^{[8]}$ 。地震保险是基于地震 风险和地震损失为基础的研究, 有必要将之与已有 地震风险和地震损失研究进行比较, 了解地震保险 对于基础研究的应用和发展情况, 寻找未来可能的 突破点。同时, 也有必要与其他巨灾保险对比, 了 解巨灾保险的总体发展。

因此本文使用 CiteSpace ${ }^{[9]}$ 对 1980-2018 年的 “地震保险”、“其他巨灾保险”、“地震风险和 损失” 三大研究主题的研究文献进行分析, 展示研 究领域的热点趋势和主题演进, 对比研究差异, 寻 找未来研究突破点。

\section{2. 数据来源与方法}

CiteSpace 软件 ${ }^{[9]}$ 是文献热点趋势和演进研究软 件, 目前国内外学者已应用此方法开展多研究领域 的分析, 本文使用此软件对 “地震保险”、“其他 巨灾保险” 和 “地震风险和损失” 三大研究主题的 研究文献开展关键词共性分析和时间演进分析, 研 究目标的热点趋势和主题演进。

本文指定检索范围为 CNKI 期刊数据库中的 “SCI 来源期刊”、“EI 来源期刊”、“核心期 刊” 和 “CSSCI”。检索时间段为 “1990 年-2018 年” 数据检索时间为 2018 年 9 月 28 日。

地震保险相关内容的检索条件为标题或关键词 为 “地震” 和 “保险”。得到检索文献共 93 条。

其他巨灾保险检索条件为标题或关键词为 “洪 水” 和 “保险” , 标题或关键词为 “台风” 和 “保 险”, 标题或关键词为 “巨灾” 和 “保险” 且不含 “地震”, 得到检索文献共 358 条。

地震风险和地震损失方面的文献检索条件为标 题或关键词为 “地震” 和 “风险”, “地震” 和 “损失”，得到检索文献共 367 条。
表 1 三大研究主题检索条件和检索结果

\begin{tabular}{|c|c|c|}
\hline 研究主题 & 检索方式 & $\begin{array}{l}\text { 文献 } \\
\text { 数目 }\end{array}$ \\
\hline 地震保险 & $\begin{array}{l}\text { 关键词: 地震 并含 保险 } \\
\text { 或篇名: 地震 并含 保险 }\end{array}$ & 93 篇 \\
\hline \multirow[t]{3}{*}{$\begin{array}{c}\text { 其他巨灾保 } \\
\text { 险 }\end{array}$} & $\begin{array}{l}\text { 关键词: 台风 并含 保险 } \\
\text { 或篇名: 台风 并含 保险 } \\
\text { 且关键词和篇名不含 地震 }\end{array}$ & 358 篇 \\
\hline & $\begin{array}{l}\text { 关键词: 洪水 并含 保险 } \\
\text { 或篇名: 洪水 并含 保险 } \\
\text { 且关键词和篇名不含 地震 }\end{array}$ & \\
\hline & $\begin{array}{l}\text { 关键词: 巨灾 并含 保险 } \\
\text { 或篇名: 巨灾 并含 保险 } \\
\text { 且关键词和篇名不含 地震 }\end{array}$ & \\
\hline \multirow[t]{2}{*}{$\begin{array}{l}\text { 地震风险和 } \\
\text { 地震损失 }\end{array}$} & $\begin{array}{l}\text { 关键词: 地震 并含 风险 } \\
\text { 或篇名: 地震 并含 风险 }\end{array}$ & 367 篇 \\
\hline & $\begin{array}{l}\text { 关键词: 地震 并含 损失 } \\
\text { 或篇名: 地震 并含 损失 }\end{array}$ & \\
\hline
\end{tabular}

\section{3. 结果和分析}

\section{1. 关键词共现分析}

关键词是对文章主题的高度凝练, 本文首先对 所有文献的关键词频率进行分析, 统计三大研究主 题文献的关键词频率。表 2 给出三大研究主题文献 的主要关键词对比 (为便于研究, 去掉了与主题相 同、前后重复和无意义的关键词）。表格中可以看 出, 地震保险研究主要围绕地震保险制度、地震风 险度量、使用金融方法进行巨灾风险转移、再保险 对巨灾风险的处理、国外已有经验、费率厘定等内 容。其它巨灾保险研究主要围绕再保险风险分散方 式、制度、建立巨灾保险基金、农业巨灾、债券方 式转移风险、美国已有经验。而地震风险和损失研 究主要围绕风险和损失的评估、各种评估方法和地 震的易损性、危险性、不确定性。

表 2 三大主题文献的关键词前十名统计

\begin{tabular}{|c|c|c|c|}
\hline $\begin{array}{l}\text { 排 } \\
\text { 名 }\end{array}$ & 地震保险 ${ }^{a}$ & 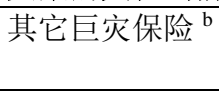 & $\begin{array}{l}\text { 地震风险 } \\
\text { 和损失 }^{c}\end{array}$ \\
\hline 1 & 地震保险制度 & 巨灾风险 & 风险评估 \\
\hline 2 & 巨灾风险 & 再保险 & 损失评估 \\
\hline 3 & 地震保险基金 & 巨灾保险制度 & 地震保险 \\
\hline 4 & 地震风险 & 巨灾保险基金 & 地震易损性 \\
\hline 5 & 再保险 & 农业巨灾保险 & 巨灾风险 \\
\hline 6 & 日本 & 巨灾风险管理 & 增量动力分析 \\
\hline 7 & 风险管理 & 巨灾债券 & 评估方法 \\
\hline 8 & 保险费率 & 分保 & 层次分析法 \\
\hline 9 & 保险基金 & 美国 & 地震危险性 \\
\hline 10 & 巨灾保险制度 & 风险分散 & 不确定性 \\
\hline
\end{tabular}


a 去掉了地震保险、灾害保险、地震灾害、地震、巨灾保 险、中国人民保险公司、人保公司、保险业。

b 去掉了巨灾保险、洪水保险、农业保险、巨灾、防洪保 险、保险、保险制度、灾害保险、保险业、洪水风险、赔 款、巨灾损失、洪水灾害、再保险人。

c 去掉了地震、地震灾害、汶川地震、评估、风险、地震 风险、风险分析、直接经济损失、经济损失、地震损失、 风险评价、损失、地震灾害损失、易损性、滑坡、中国大 陆、间接经济损失、风险感知。

使用 CiteSpace 对关键词的进行共现和聚类分 析, 可以确定相关研究的关键词联系, 找到核心研 究内容。图 1、图 2、图 3 分别给出地震保险、其它 巨灾保险、地震风险和损失三大主题的关键词聚类 分析。

从图中可以看出, 地震保险研究主要围绕地震 保险制度、巨灾基金、免赔额和保费、包头市的案 例、风险转移研究。

其它巨灾保险主要围绕农业巨灾、洪水保险和 洪水保险基金、巨灾保险制度、巨灾债券开展研 究。而地震风险和损失研究主要围绕风险评价或评 估、地震易损性、地震损失和汶川地震的风险和损 失研究几个方面。

\section{2. 关键词时间线分析}

时间线可以展示关键词的时间演进。图 4、图 5、图 6 分别是地震保险主题文献的关键词共现时间 线图、其它巨灾保险主题文献的关键词共现时间线 图和地震风险损失主题文献的关键词共现时间线 图。

早在 1992 年国务院就通过国办发文的形式明确 地震保险的地位, 指出 “开展地震保险是实现社会 互助、减轻国家财政负担、提高抗震救灾能力的有 效途径”, 而地震保险的研究也在上世纪九十年代 初就已经存在（如图 4 所示）。1996 年丽江 7.0 级 地震造成保险公司偿付能力危机, 于是 1996 年中国 人民银行将地震作为一般财产保险的除外责任, 另 一方面中国人民银行特别召开全国地震保险座谈 会, 并资助《中国地震保险保险费率区划图编制暨 地震保险条款的制定》、《中国地震保险基金的建 立和渠道》、《中国地震再保险运作机制的研究》 三个地震保险课题, 对地震保险开展专门研究 ${ }^{[10]}$ 。 自此地震保险的相关研究和讨论逐渐展开。但 19972007 年, 地震保险相关研究保持平稳, 期间仅有少 数新增关键词出现。2008 年, 中国经历改革开放以 来最大损失的地震一一汶川地震, 它给我国人民的 财产和生命带来了极大的损失。以此事件为标志, 举国上下开始重视地震灾害及其影响, 地震保险也 随之迎来又一个研究高峰。之后地震保险研究进入 持续热烈讨论期, 研究内容和主题也不断增加和扩

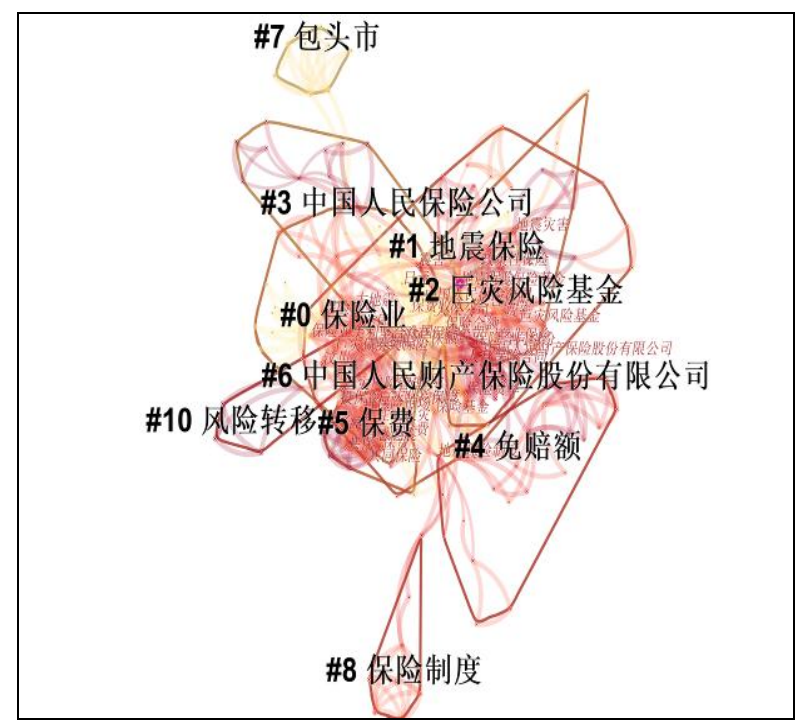

图 1 地震保险主题关键词共现聚类图

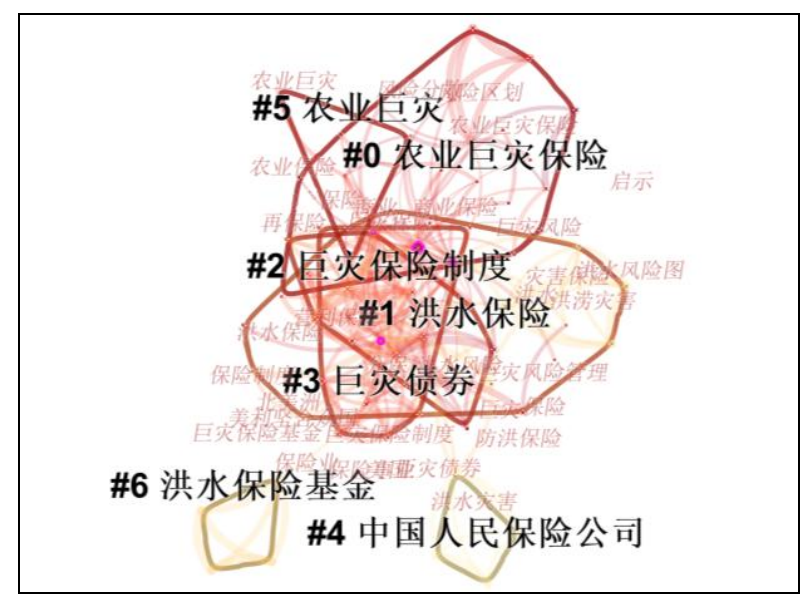

图 2 其它巨灾保险主题关键词共现聚类图

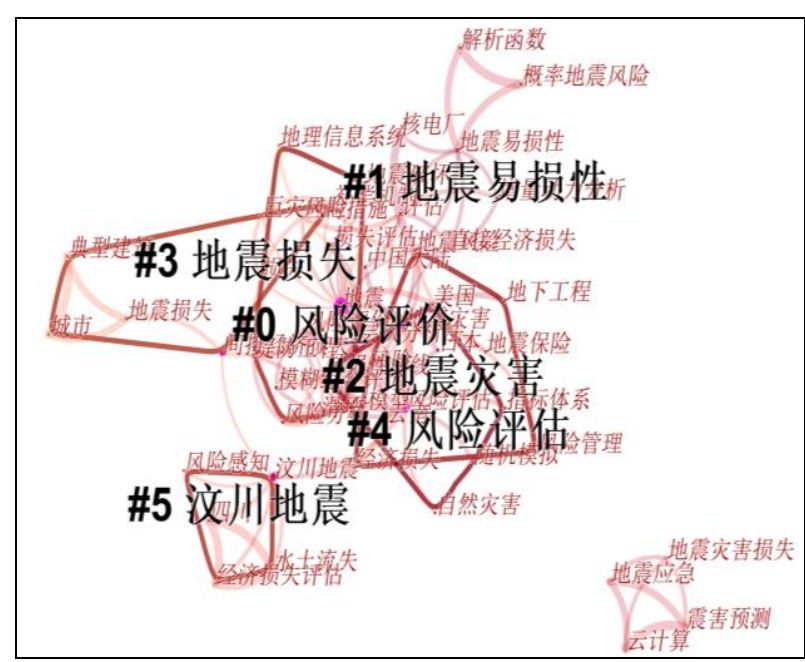

图 3 地震风险和损失研究主题关键词共现聚类图 


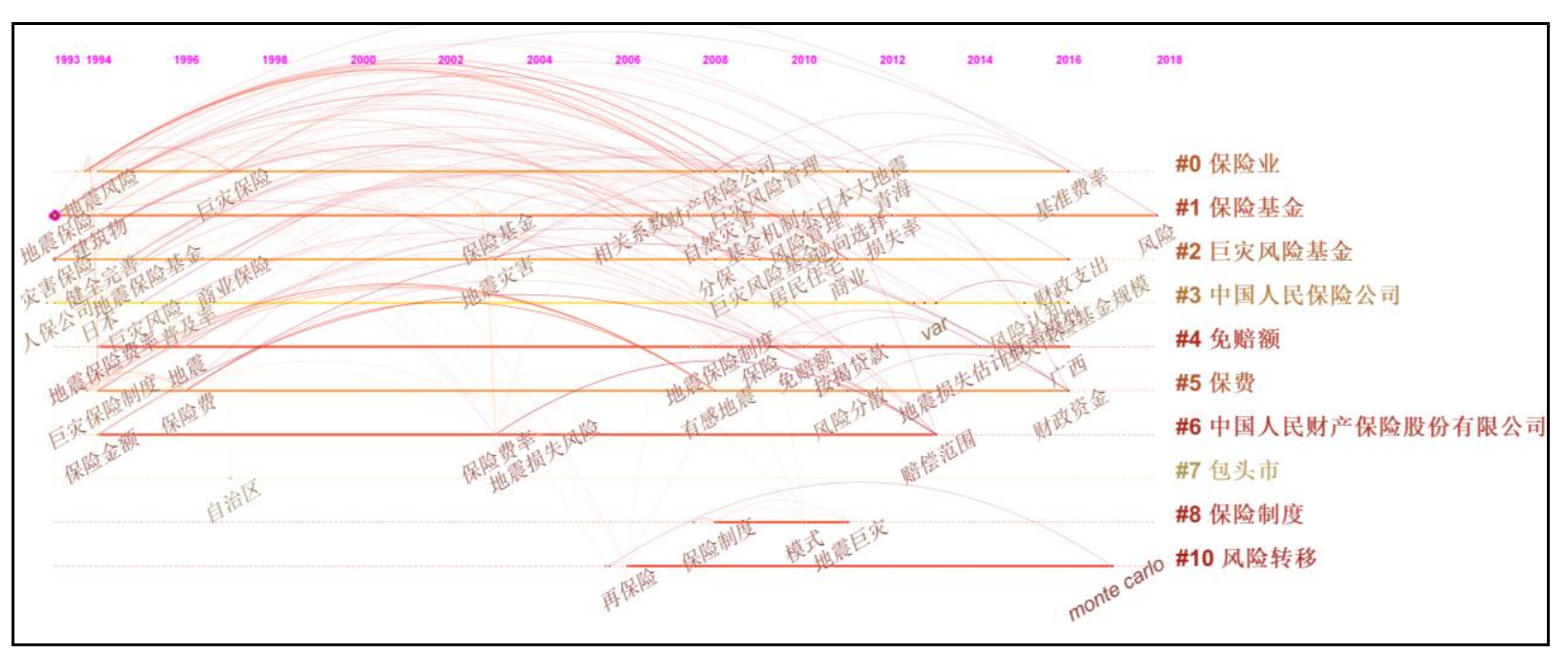

图 4 地震保险文献关键词时间线趋势图.

充, 形式从个人财产保险到对公指数保险, 风险分 散方式也扩充到了国际金融领域，模拟方法也从定 性或概率性的方式进化到如蒙特卡罗为代表的随机 模拟阶段。但从整体而言, 地震保险相关研究更多 注重于公共管理制度的研究和经济金融方面风险转 移和分担的研究, 对于工程性的技术类的研究几乎 没有。

图 5 给出其它巨灾保险文献研究的时间线。农 业巨灾保险是其它巨灾保险一直最为热烈的研究 点, 从 1994 年美国农作物保险改革一直持续到现 在。洪水保险则在 1998 年长江中下游洪水灾害的背 景下, 在 1998 年成为研究爆发点。巨灾保险制度研 究则在 1997 年开始, 而巨灾债券 1995 年美国芝加 哥交易所最先推出, 1998 年被介绍到中国学术界。 但总体而言, 其它巨灾保险相关研究同样多注重于 制度研究和风险转移和分散的研究, 对于工程性的 技术类的结合研究几乎没有。
图 6 给出地震风险和损失主题文献的关键词时 间线。地震风险和损失的研究热点主要集中在风险 评价、风险评估、易损性等方面。研究技术手段多 样, 从模糊方法到云模型再到增量动力分析, 从概 率性风险分析到解析函数法。研究内容不仅包括地 震本身, 也包括地震引起的次生灾害——如滑坡、 地震相关的堤防工程以及水土流失。研究内涵上, 不仅包括工程方面，也包括社会人文方面的风险感 知。但总体而言, 对于风险和损失的研究多集中在 技术手段, 而风险管理相关研究较少, 风险分散研 究几乎没有, 社会人文方面涉及很少, 只有汶川地 震相关的风险感知研究。

因此，本文认为地震巨灾保险和其它巨灾保险 研究具有注重制度、技术等非工程方面的研究的相 似性; 无论是地震巨灾保险还是其它巨灾保险都较 少结合工程技术。另一方面, 地震风险和损失研究 则多注重工程技术，较少结合制度、风险管理等非

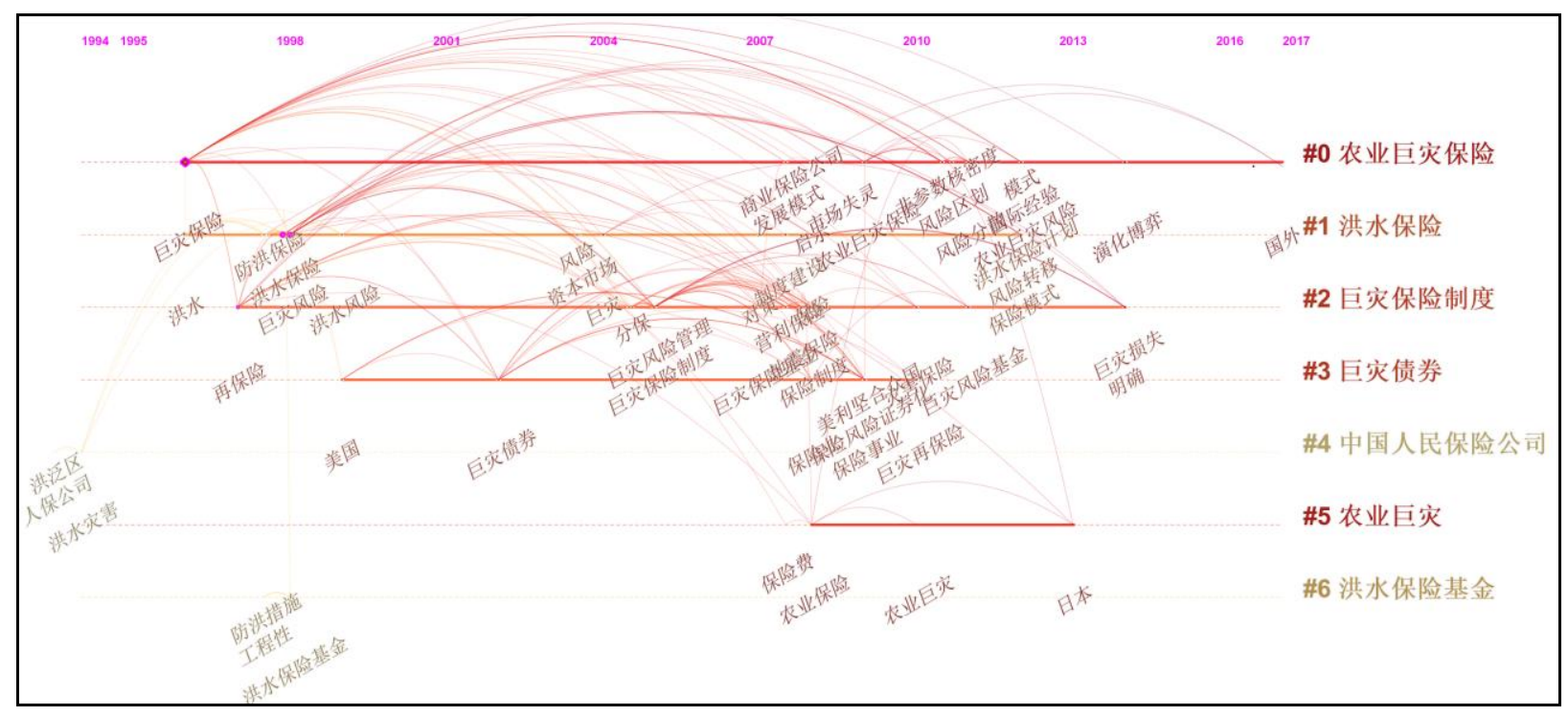

图 5 其它巨灾保险文献关键词时间线趋势图. 
工程防御手段。巨灾保险研究和地震风险损失研究 之间跨学科研究较少, 缺少融合式发展。

\section{3. 机构共现分析}

本文进一步对地震保险和地震风险损失两大主 题的研究机构之间的共现关系进行分析。图 7、图 8 分别是地震保险主题文献的研究机构共现图和地震 风险损失主题文献的研究机构共现图。

由图 7 所示, 参与地震巨灾主题的研究机构 中, 各单位联结较少, 只出现了 7 个组团的关系网 络, 并且这仅有的 7 个组团网络的联结也都比较分 散, 多为两点或三点型, 只有国科大-四川大学-同济 大学-上海财经大学关系网络是多合作, 且合作的关
系网中，也只有此关系网络是经管类学科和工程类 学科合作, 其它组团的关系网络均为单一学科合 作。

如图 8 所示, 地震风险和损失的研究也存在类 似现象。首先各个单位的交流合作不多, 只有 7 个 关系网络形成，其中只有一个关系网格是 7 点，一 个关系网格是 4 点, 且这两个多点网格还未形成闭 合网络, 各单位之间缺少交流合作。其次, 同样存 在学科割裂的现象。参与地震风险和损失研究的机 构和单位绝大多数都是理工科院校或院系，其中只 有对外经贸大学保险学院, 武汉大学经管学院和防 灾科技学院经管系三个非理工科院系。并且这个三 个非理工科院系也和其它单位缺少有效交流, 没有

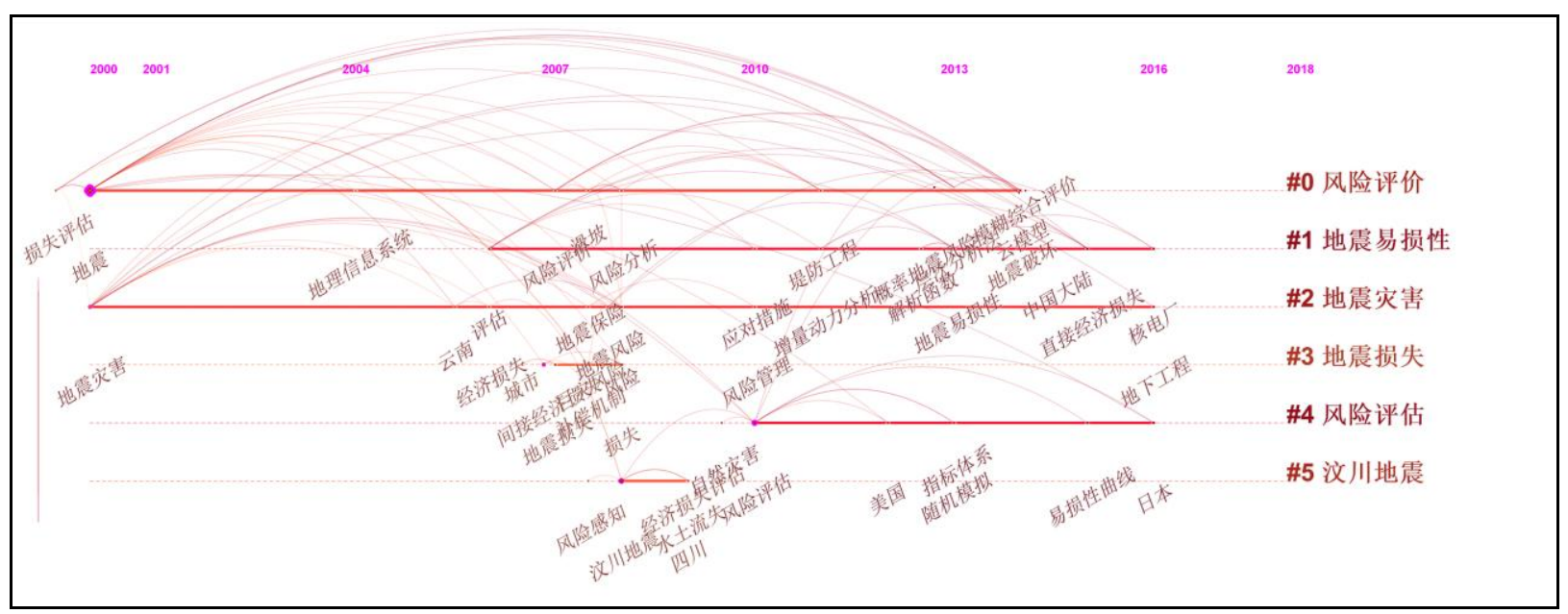

图 6 地震风险和损失文献关键词时间线趋势图.

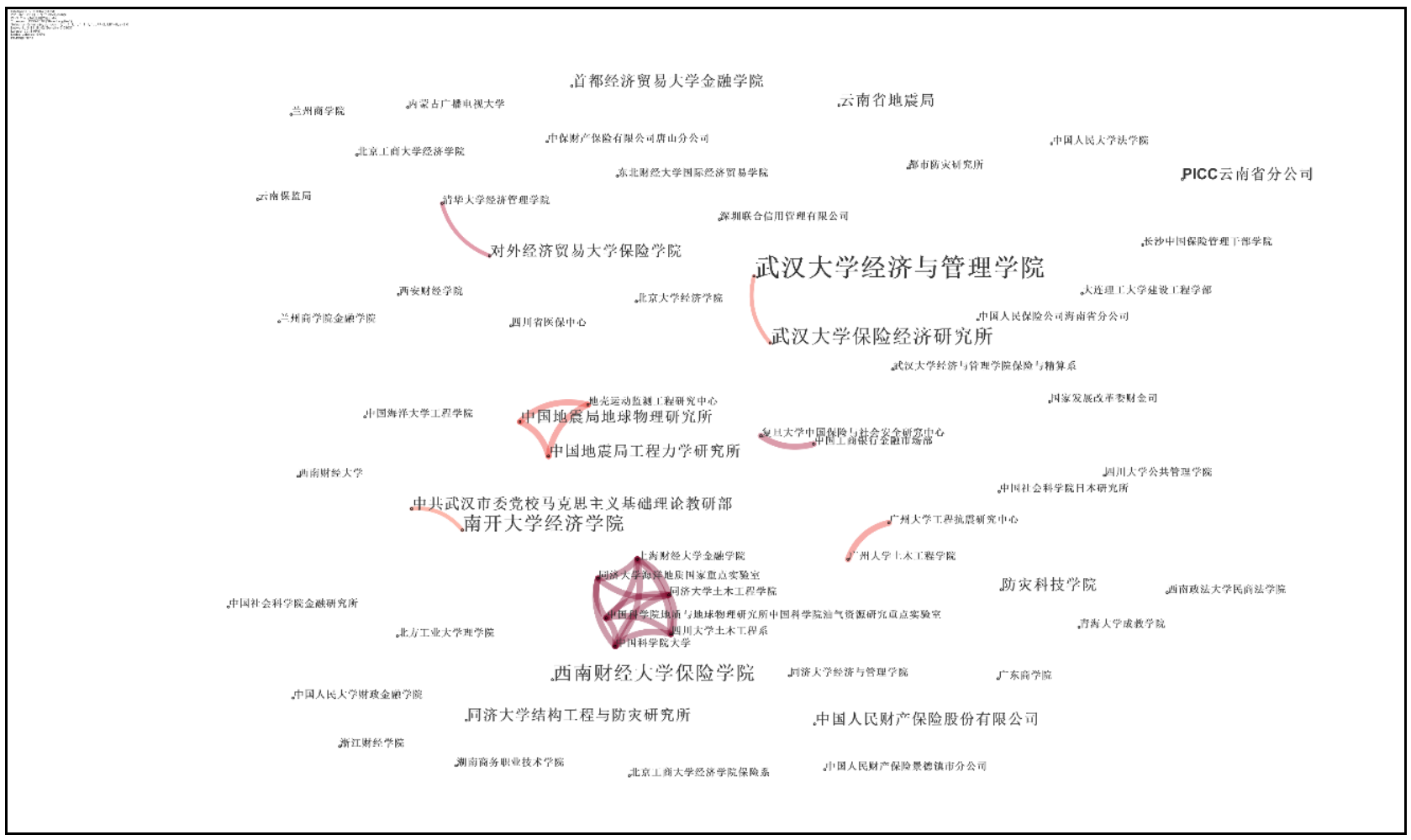

图 7 地震保险主题文献的研究机构共现图 


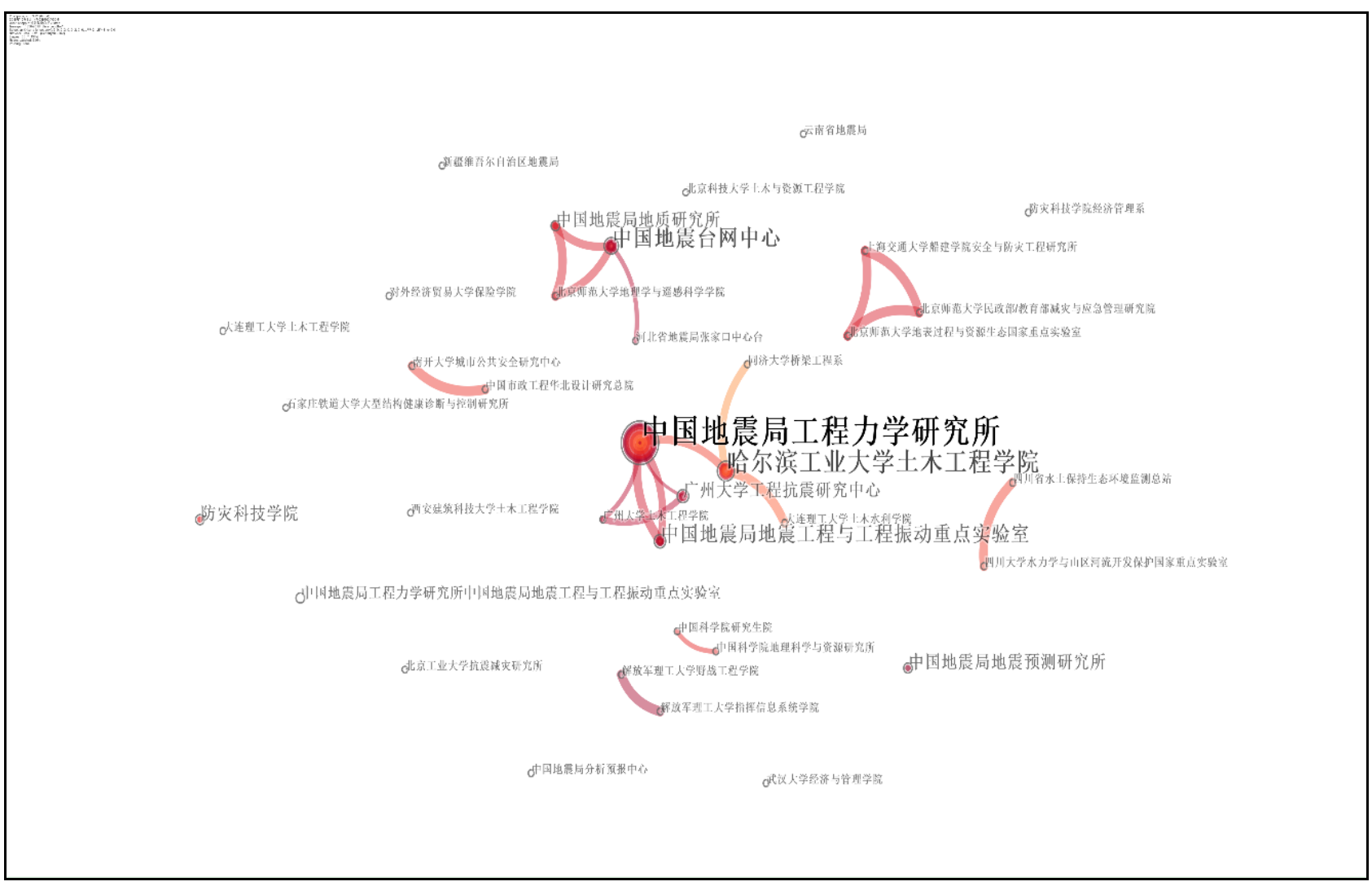

图 8 地震风险和损失主题文献的研究机构共现图.

形成联系。

因此, 本文认为地震巨灾保险和地震风险和损 失研究都存在交流合作少、学科割裂的现象。

\section{4. 结论与展望}

本文使用 CiteSpace 软件对 “地震保险”、“其 他巨灾保险”、“地震风险和损失”三大研究主题 的研究文献开展关键词共性分析和时间演进分析。

分析表明, 地震巨灾保险和其它巨灾保险研究 具有注重制度等非工程方面的研究的相似性; 无论 是地震巨灾保险还是其它巨灾保险都较少结合工程 技术。而地震风险和损失研究则多注重工程技术, 较少结合制度、风险管理等非工程防御手段。巨灾 保险研究和地震风险损失研究之间的跨学科研究较 少, 缺少融合式发展。

进一步, 地震保险和地震风险损失文献的研究 机构共现网络分析则表明, 地震巨灾保险和地震风 险和损失研究存在机构交流合作少、学科割裂的现 象。

地震是我国发生频繁且严重威胁国民安全制约 经济社会发展的自然灾害之一。为减少地震风险, 应同时设法减少暴露性和脆弱性, 增加社会㓞性,
做到真正的融合式发展，使工程措施和非工程措施 相辅相成, 降低全社会的地震风险。

\section{5. 致谢}

本项目由中国地震局地球物理研究所基本科研 业务经费资助（项目编号 DQJB17C10）。

This project is sponsored by: Special Fund of the Institute of Geophysics, China Earthquake Administration (Grant Number DQJB17C10).

\section{References}

[1] 胡聿贤. 地震工程学(第二版). 北京: 地震出版社, 2006 . $\mathrm{Y}$. Hu, Earthquake Enginering. 2nd Edition. (Earthquake Publish Company, Beijing, 2006)

[2] 李善邦.中国地震. 北京:地震出版社, 1981 .

S. Li, Earthquake in China. (Earthquake Publish Company, Beijing, 1981)

[3] UNISDR, Global assessment report on disaster risk reduction: risk and poverty in a changing climate. (UNISDR, Geneva, 2009)

[4] USGS, Historic World Earthquakes. (2004) http://earthquake.usgs.gov/earthquakes/world/historihis. php. 
[5] T. Utsu, A List of Deadly Earthquakes in The World: 1500-2000, in International Handbook of Earthquake and Engineering Seismology, (2002) 691-717.

[6] NOAA, The National Oceanic and Atmospheric Administration (NOAA) Online Catalog. (2010) Http://www.ngdc.noaa.gov/hazard/earthqk.shtml.

[7] 郑通彦,李洋,侯建盛等,2008 年中国大陆地震灾害损 失述评,灾害学,25(2)(2010)112-118.

T. Zheng, Y. Li, J. Hou, H. Mi, Review on earthquake disaster loss in Chiness mainland in 2008. Journal of Catastrophology. 25(2)(2010)112-118.
[8] P. Grossi, H. Kunreuther, catastrophe modeling: a new approach to managing risk. (Springer, New York, 2005)

[9] C. Chen, CiteSpace. http://cluster.cis.drexel.edu/ cchen/citespace/.

[10] 邹其嘉, 顾建华, 我国地震保险的研究与展望, 城市与 减灾,(2),(2001),8-11.

Q. Zou, J. Gu, Research and prospect of earthquake insurance in China. Cities and Disaster Reduction, (2),(2001),8-11. 